\title{
Recent Investigations of the Mechanics of Cavitation and Cavitation Damage
}

\author{
BY ROBERT T. KNAPP, ${ }^{2}$ PASADENA, CALIF.
}

This paper describes water-tunnel investigations into the mechanics of "fixed"-type cavitation and into the probable mechanism through which this type causes material damage. High-speed motion pictures were used to study the cavity mechanics, and indications of the damage pattern were obtained by measuring the pitting rate on soft aluminum test specimens. Information was obtained on the frequency and intensity of the damaging blows, the distribution of damage in relation to the area covered by the cavitation, and the variation of the intensity of cavitation with velocity.

\section{ObJectivie of Study}

$\mathrm{T}$ HE purpose of this paper is to present additional experimental evidence on the mechanics of cavitation, with particular emphasis on those phases responsible for the production of damage on the adjacent solid guiding surface. In the past $(1)^{2}$ most of the work done in this field has been concentrated along two lines: (a) Investigations of the general mechanics of the cavitation process $(2,3,4,5,6) ;(b)$ a study of the relative resistance of materials to cavitation damage $(7,8,9)$. The objectives of most of the studies of the cavitation process have been the clarification of the behavior of the cavities in the liquid, and the development of analytical expressions to describe flow with cavitation. On the other hand, the investigations of the relative resistance of materials to cavitation damage have been concentrated largely on the behavior of the materials when subjected to some standardized type of cavitation. In addition to such empirical determinations of the relative resistance, considerable work has been done on the effects of the cavitation attack on the physical properties of the material. Thus the microstructure of the material has been studied to determine the physical changes produced by the cavitation which eventually lead to the removal of elements of the material from the surface. However, as yet there is only a partial understanding of the detailed mechanics by which the cavitation process in the liquid produces forces of sufficient intensity to cause physical damage on the guiding surface.

In 1917 Lord Rayleigh (10) presented a simple analysis of the collapse of an empty cavity in an ideal liquid which demonstrated that very high forces would be produced at the instant of collapse. However, it is only comparatively recent that the development of high-speed cinematography has made it possible to obtain experimental evidence showing that traveling cavitation voids collapse in good agreement with Rayleigh's analysis. This confirmation suggested the possibility of correlating the mechanics

1 Professor of Hydraulic Engineering, Hydrodynamics Laboratory, California Institute of Technology. Life Mem. ASME.

2 Numbers in parentheses refer to the Bibliography at the end of the paper.

Contributed by the Hydraulic Division and presented at the Annual Meeting, New York, N. Y., November 28-December 3, 1954, of The American Society of Mechanical Engineers.

Note: Statements and opinions advanced in papers are to be understood as individual expressions of their authors and not those of the Society. Manuscript received at ASME Headquarters, August 17 1954 Paper No 54-A-106.

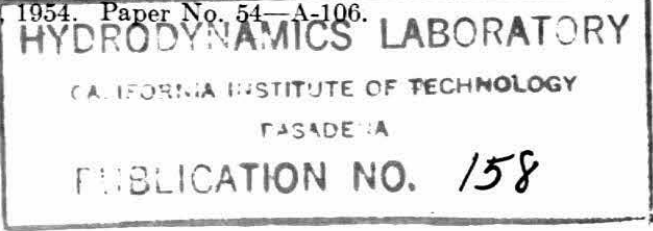

of the cavitation process with the mechanies of surface damage. Study along these lines soon brought to light another difficulty. The agreement obtained between the Rayleigh analysis and the laboratory experiments was for individual traveling cavities. However, it developed that the more general type of cavitation occurring in hydraulic equipment is of the fixed-cavity variety. In this type of cavitation the main flow leaves the guiding surface and follows a free trajectory which usually returns to the surface at some downstream point. The fixed cavity is the space between the solid guiding surface and the free-liquid surface.

On preliminary examination this type of cavitation appears to be quite different from that caused by traveling cavities, since there seems to be no sudden collapse. If this were actually the case, the Rayleigh analysis would be inapplicable; thus some other mechanism would be required to explain the production of forces of high enough intensity to cause damage.

Since it is difficult to distinguish between the two types of cavitation with the unaided eye, there is only a little evidence to show which type occurs in given specific cases. However, the available evidence shows, reasonably conclusively, that damage is also produced by fixed-type cavitation. Hence it seemed necessary to make a more detailed investigation of the fixedcavity mechanics to determine, if possible, the origin of the highintensity forces that produce damage. This paper will be restricted to the discussion of laboratory experiments on fixed cavities and the evaluation of the results obtained.

\section{Description of Experiments}

Two types of experiments have been made. The first consists of a series of high-speed motion pictures to permit the study of the detailed mechanics of fixed-type cavitation on a simple body shape $(11,12)$. Various photographic reduction ratios and camera positions resulted in both general views of the entire cavitation region and detailed studies of the major features. Most of the work was done on a body of revolution consisting of a cylindrical center section, a hemispherical nose, and a long ogival afterbody. Attention was concentrated upon cavities which did not extend beyond the downstream end of the cylindrical section. These records showed that an irregular cyclic process was going on within the cavity, and enough indirect evidence was obtained to give a general idea of the nature of this process. However, it seemed desirable to confirm this with more direct evidence. Therefore a series of three geometrically similar two-dimensional bodies was constructed with semicircular noses, parallel midsections, and ogival afterbodies. Their mid-section thicknesses of 1,2 , and 4 in. were chosen to see if any significant differences in the flow patterns could be observed. Each had a span of 2 in., with end shields of clear plastic. High-speed motion pictures were taken, both in profile and in plan, for various cavity lengths at flow velocities of 25 and $50 \mathrm{fps}$. This was the highest velocity usable with the plastic end shields. However, velocities up to $100 \mathrm{fps}$ were used with the bodies of revolution.

The second set of experiments was made on geometrically identical bodies of revolution, namely, a 2-in-diam cylindrical mid-section with a hemispherical nose and an ogive afterbody. However, in this series, 3 in. of the cylindrical center section, on which the cavitation occurs, was constructed of a material of 
relatively low resistance to cavitation damage, since the major objective was the study of the development of damage and its relation to the physical characteristics of the cavitation. Two types of information were collected: (a) Relatively large-scale bigh-speed motion pictures of the cavitation actually occurring while damage was taking place; and $(b)$ repetitive photomicrographs of representative areas along the length of the test section. These photomicrographs were closely spaced to cover the entire length of the cavity and also the adjacent downstream section. A technique was developed which made it possible to secure these photographs without removing the test body from the tunnel and without lowering the water surface enough to expose any portion of the body to the air. Thus the critical center sections were not handled during the entire run. As a matter of fact, they were not even touched. Therefore all changes in the successive photomicrographs were due to the cavitation.

Test Sections. The first test sections were made of freemachining brass which is known to have a relatively low resistance to cavitation damage, and is usually used as a calibration standard for the magnetostriction type of testing equipment. These test sections were run in the high-speed water tunnel for maximum periods of $25 \mathrm{hr}$ at a flow velocity of $90 \mathrm{fps}$, and with cavity lengths of either 1 or 2 in. The photomicrographs showed definite evidence of damage, increasing with time. However, the damage rate was considered to be too low since the time required to test a single specimen was quite long and the tunnel operation, especially at this maximum permissible sustained speed, relatively expensive. It was felt that a satisfactory material should have the following properties: $(a)$ It should show appreciable microscopic damage in an hour or less; $(b)$ different lots obtained in the open market should all have the same physical properties; (c) it should be a metal, since most important cases of cavitation damage involve metallic surfaces; $(d)$ its corrosion resistance should be relatively high compared to its cavitation resistance to minimize confusion between these two effects.

Commercially pure annealed aluminum conforms closely to these requirements. It is more readily damaged by cavitation than free-machining brass; it can be annealed readily in a simple furnace, after machining, to bring it to a standard and reproducible physical condition. Annealed 2S-F aluminum was therefore selected for trial. Since even before annealing, this material is very soft and therefore difficult to polish satisfactorily, a process was developed in the instrument shop which produced a satisfactorily smooth surface with a cutting tool. It was also found that the low temperatures required for subsequent full anneal did not change the surface finish.

Procedure for Typical Experiment. The procedure for a typical experiment was as follows: The test body was installed in the tunnel on a sting support and the tunnel was filled until the water was approximately $1 \mathrm{in}$. above the body. Then a microscope support and traverse mechanism were fastened to the open access window directly above the aluminum mid-section. A low-power microscope with camera attachment was used with a dipping cone so that the objective could be immersed to the proper depth. A Heine Ultropak illuminator was used, which is a variation of the vertical type. Photomicrographs were taken at a magnification of 30 diam with a field coverage of approximately 0.2 in. Twelve photographs were taken along the 3 -in. test section with fields slightly overlapped to give complete coverage of the critical damage zone and 50 per cent coverage for the remainder of the section. Next, the tunnel was closed, filled, and the test run was made at the selected velocity, cavity length, and period. The tunnel was then stopped, the window opened, and another set of photomicrographs was taken of the identical areas.

Objectives of Test Runs. Since these runs were planned to supple- ment the investigation of the fixed-cavity mechanics, the main objectives were to study the variation in damage along the length of the test section in relation to the position of the fixed cavity and to attempt to correlate the type of damage observed with the cavity mechanics. A further objective was to investigate the amount of damage as a function of the time of operation under cavitating conditions. The final objective was to attempt to secure preliminary information about the variation of the intensity of cavitation with changes in the velocity of the flow.

All tests except those used to explore the final objective were made with a tunnel velocity of $90 \mathrm{fps}$. Cavity lengths of 1 and 2 in. were studied, with separate aluminum test sections. A set of runs, starting with a period of $10 \mathrm{~min}$ and approximately doubling the length of each successive run, was made on a single test section until a total test period of $71 / 2 \mathrm{hr}$ had been obtained. Complete sets of 12 photomicrographs were taken $(a)$ before the initial run, $(b)$ between each successive run, $(c)$ at the completion of the final test period.

During one run a high-speed motion picture was taken of the cavity at an exposure rate of 10,000 frames per sec. A similar set of runs was made on another aluminum test section for a cumulative time of $24 \mathrm{hr}$, and two otherwise identical sets were made using a 2-in. cavity length in place of the 1-in., the first one for a cumulative time of $12 \mathrm{hr}$ and the second one for $40 \mathrm{~min}$. This last set started with a $21 /{ }_{2}$-min period because considerable pitting had been observed to take place in the initial $10-\mathrm{min}$ period of the first three sets. A number of pits formed even in $2^{1 / 2} \mathrm{~min}$; therefore another series of tests was run starting with a much shorter period. In this series a new test section was used for each run so that the damage on the entire section could be studied in more detail at a later time. It was found that the shortest practicable test period was 30 sec. Beginning with this, separate test sections were run for periods of $1 \frac{1}{4}, 2^{1 / 2}, 5,10$, and $20 \mathrm{~min}$.

The tests made to explore the final objective, i.e., the effect of variations in velocity on the intensity of cavitation, also employed separate test sections for each run. Since these tests were not planned until a preliminary analysis had been made of the results of the preceding studies, it was possible to take advantage of the experimental fact that for given conditions the rate of pitting did not vary with the length of the run. Thus the length of run was varied for the different velocities to obtain satisfactory counting densities of the pits. The runs were started at the maximum speed of the tunnel-100 fps; the next runs were made at $90 \mathrm{fps}$, and the third at $77^{1 / 2} \mathrm{fps}$. The run lengths were all $10 \mathrm{~min}$. The rate of pitting observed on the test section from the latter run was so low that smaller steps in speed and longer times were selected for the subsequent tests, i.e., 71 and $63 \mathrm{fps}$, each for 20 min. The final run was made at $59 \mathrm{fps}$ using three times the initial test period of $10 \mathrm{~min}$. Under these conditions the pitting rate was so low that this preliminary series of tests was terminated.

\section{Experimental Results}

Mechanics of Fixed-Type Cavitation. This discussion is based upon observations of cavitation on both the bodies of revolution and the two-dimensional bodies. All of these bodies had the same geometrical cross section, i.e., a semicircular nose, a parallel mid-section, and an ogive afterbody.

The physical concept of fixed cavitation is that such a cavity is formed by the breaking away of the flow from the guiding surface at a low-pressure point, usually assumed to be the point at which the pressure on the surface has fallen to the vapor pressure of the liquid. From this point the stream follows a trajectory determined by the pressure field, and usually recontacts the guiding surface at some distance downstream from the breakaway point. The fixed cavity is the space existing between the guiding surface and the free surface of the flow. The high-speed motion 
pictures show that this cavity is fixed only in a statistical sense. Steady flow conditions are not established; instead, a continuous but somewhat irregular cyclical process develops. In each cycle three phases can be distinguished: (a) Formation and growth, $(b)$ filling, and $(c)$ breakoff.

For a given set of flow conditions, the maximum length of cycle occurs when it starts with no residual flow cavity, grows to the characteristic length, fills completely, and breaks away from the body. The breakoff time is negligible compared with that of the other phases. Shorter periods occur when breakoff comes after only partial filling, or when growth commences with a large residual cavity. Early breakoff seems to be associated with disturbances within the cavity rather than within the main flow. The stream of liquid that fills the cavity is the re-entrant flow which forms in the high-pressure area at the downstream end of the cavity. This re-entrant flow performs the necessary function of supplying the reaction force which turns the remainder of the flow downstream and parallel to the guiding surface. The upstream velocity of this re-entrant flow is proportional to the velocity at the free surface of the cavity and would be of equal magnitude if it were not for skin friction. However, since the re-entrant flow is a relatively thin sheet, skin friction is a large effect

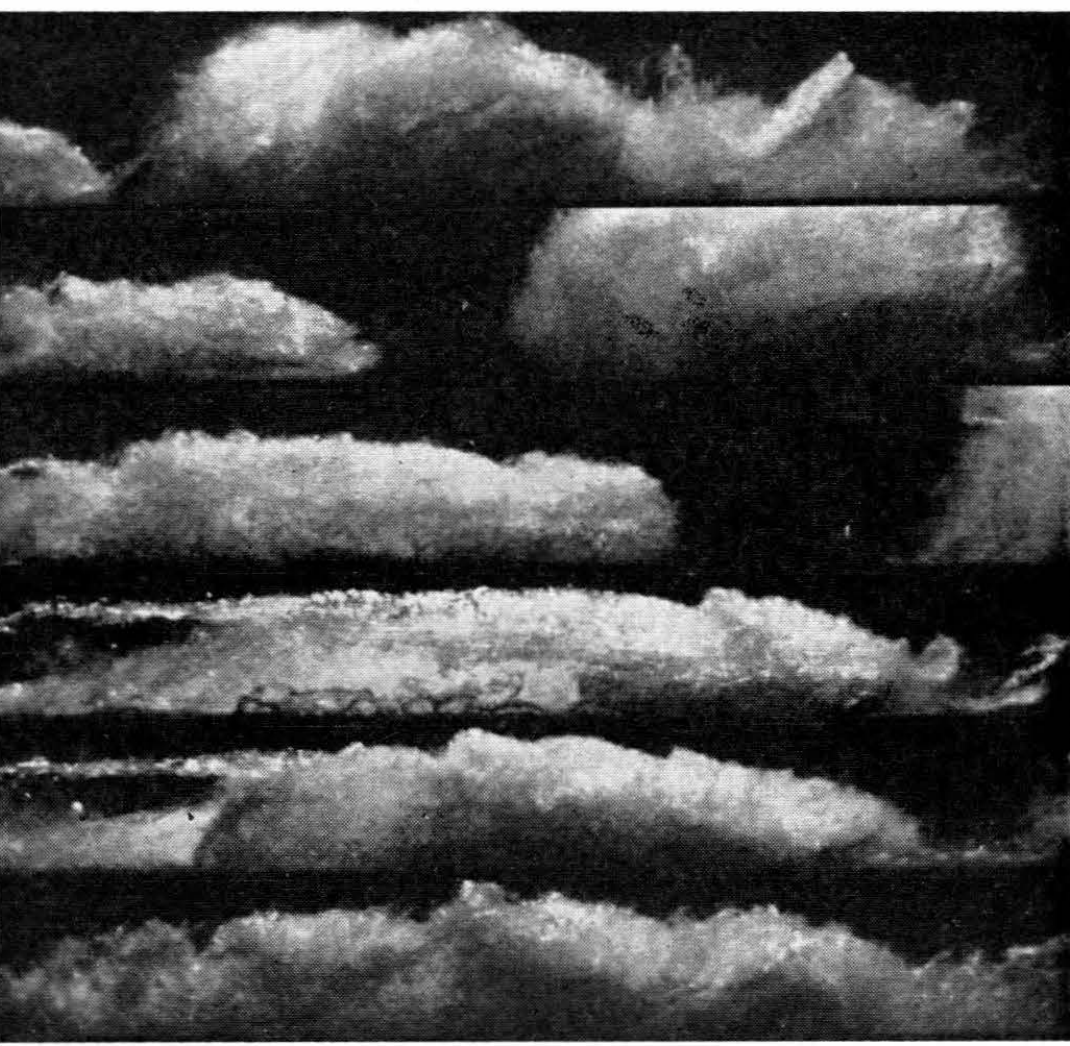
and rapidly reduces this upstream velocity.

Fig. 1 is a series of motion-picture frames showing the cavity growth, filling, and breakoff. The re-entrant flow is seen clearly in the fourth and fifth frame. The last frame shows the breakup of the surface which occurs at complete filling. As indicated in Fig. 2, the liquid in this re-entrant flow consists largely of the fluid that was adjacent to the free surface of the cavity. This is a significant factor in the explanation of the observed pattern of cavitation damage. Another significant detail shown in Fig. 2 is the stagnation zone which divides the re-entrant flow from the main flow. This is a zone of high relative pressure, since it is a full velocity head greater than the static pressure in the undisturbed flow.

The free surface of the cavity appears to be covered with a multitude of small cavities. Close inspection of the photographs shows that these small cavities first appear and grow rapidly to their ultimate size at the upper curved end of the main fixed cavity. After this initial growth, they move at substantially free-stream velocity and maintain a constant diameter until they reach the downstream end of the cavity, at which point they disappear.

If the behavior of the majority of these small traveling cavities is considered in conjunction with the cyclic process of cavity formation, filling, and breakoff, a rational picture develops. Most of these cavities, being small and apparently very close to the interface, will be in the re-entrant flow. The smaller ones will pass close to the stagnation point and larger ones may be swept into it. Some of the largest project into the main flow just outside of the stagnation streamline. However, all of these flow layers pass through the high-pressure area, and therefore the vast majority of these small entrained cavities must be collapsed completely. In the photographs a nebulous cloud flows away from the downstream end of the cavity during the filling part of the cycle. This may consist of minute traces of noncondensable

Fig. 1 Cavity Formation and Re-Entrant Flow

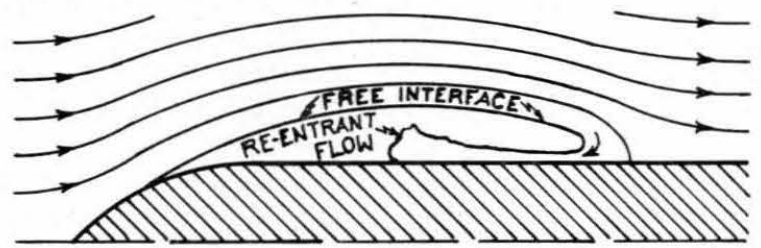

Fig. 2 Fixed-Cavity Flow

gas left after the condensation of the vapor which fills the voids.

Cause of Damage From Fixed-Type Cavitation. This description of the mechanics of fixed-type cavitation offers a simple explanation of the production of damage since the collapse of these small traveling cavities is concentrated into a narrow zone adjacent to the stagnation line. Furthermore, the pressures available to produce collapse are very much greater than the free-stream pressures; hence although the cavities are initially small, the final collapse velocities may well produce pressures high enough to exceed the yield point of the guiding surface and thus cause damage. In this type of flow the maximum pressure zone is on the surface and the direction of motion of the traveling cavities is toward the surface so that collapse may take place practically on the surface. Another important point is that, although the stagnation point remains relatively fixed at the end of the fully developed cavity for a major fraction of each cycle, nevertheless during the development phase, this stagnation point sweeps the entire length of the cavity. Owing to the many partial breakoffs that are observed, the relative time of dwell of the stagnation zone is a minimum at the upper end of the cavity and increases to a maximum at the equilibrium length. After breakoff the filled cavity acts as a foreign body over which the flow has to pass. It starts from rest and is accelerated to the stream velocity partly by the friction 
force of the main stream on this slug of liquid, but also by a direct pressure force due to the impingement of the flow that passes over the newly formed cavity, Fig. 1. This impingement explains the rapid increase in thickness of the upstream end of the detached filled cavity. This process continues well below the normal downstream end of the fixed cavity, and since this impingement produces a stagnation point on or near the guiding surface, traveling zones of high pressure must sweep periodically along the body for a considerable distance downstream from the equilibrium length of cavity. Such high-pressure zones will collapse any traveling cavities which have escaped the first high-pressure area or which are rebounds of previously collapsed cavities.

Effect of Change of Velocity on Cavity Cycle. There seems to be a characteristic change in the cavity mechanics with change in velocity. The strong cyclical behavior with nearly complete filling of the cavity on each cycle seems to be associated with the higher velocities, i.e., from $\mathbf{5 0}$ fps up. For the lower velocities, the flow appears steadier and seems to have a more continuous entrainment at the rear. Detailed examination of this entrainment, however, shows that it is not uniform, but pulsating. It seems probable that for these lower velocities the re-entrant flow may not have sufficient energy to penetrate upstream until the cavity is completely filled. Instead, it may contact the free surface near the lower end of the cavity, and such contact may result in premature breakoff and regrowth. Since the amount of filling is small the corresponding frequency is relatively high, which makes the entrainment appear more nearly continuous. These last remarks describe the variation in behavior of cavitation on the 2-in-diam models used in the water tunnel. It seems probable that for other sizes the velocity range for corresponding changes would vary since this behavior may be affected by both the body size and the properties of the liquid.

Since the taking rate of the high-speed motion pictures is controlled by a very stable oscillator, \pm 0.02 per cent, these records can be used to determine velocities, accelerations, and other time-dependent quantities. One time of interest is that required to fill the cavity. In determining this time, care was taken to avoid records which showed partial breakoffs. The volume of the fully developed cavity is also obtained from the photographs since the length, height, and profile can all be measured. Knowing the cavity volume, the time between breakoffs, and the re-entrant velocity, it is possible to compute the thickness of the re-entrant flow. If a frictionless fluid is assumed, the speed of the re-entrant flow will be the same as that along the free interface, i.e., the stream velocity multiplied by $(1+K)^{1 / 2}$. For the real fluid it is probable that the velocity of the re-entrant flow is more nearly that of the undisturbed stream.

It is also possible to estimate the thickness of the re-entrant flow from independent considerations. Gilbarg (13) has shown that when a cavity is formed downstream from a symmetrical curved obstacle, the drag on the obstacle is equal to the change in momentum of the liquid which forms the re-entrant jet. This relationship can be used to calculate the cross section of the re-entrant jet, since its velocity is known. In the experiments under discussion the cavity does not envelop the body, but collapses on the cylindrical center section. However, the basic nature of the phenomenon is unchanged. Thus it can be assumed that the increase in the nose drag caused by the presence of the cavity is equal to the change in the momentum of the re-entrant flow.

The experimental measurements of Rouse (14) have shown that upstream from the point at which the cavity springs clear, the pressure distribution on the nose of a body with cylindrical midsection is unaffected by the presence of the cavity. These experiments cover a wide range of nose shapes from sharpcornered flat noses to ogives, ellipsoids, and half-bodies. Hence the increase in the nose drag due to the cavity can be calculated from the pressure distribution for noncavitating flow, since the cavity separates from the nose at the point where the ratio of the negative pressure to the velocity pressure equals the observed $K$ for the given cavity lengths.

Fig. 3 shows the distribution of the axial component of the drag force on a hemispherical nose. This was plotted from the data of Rouse. The total area under the curve, multiplied by $2 \pi$, is the drag coefficient $C_{d}$. The shaded area is the increase in drag coefficient due to the presence of a cavity having a $K$ of 0.378 . This corresponds to one of the tunnel runs for which good highspeed motion pictures were available. These pictures were used to determine both the average longitudinal cross section of the cavity and the cycle time between major breakoffs. This completes the information required to estimate the re-entrant

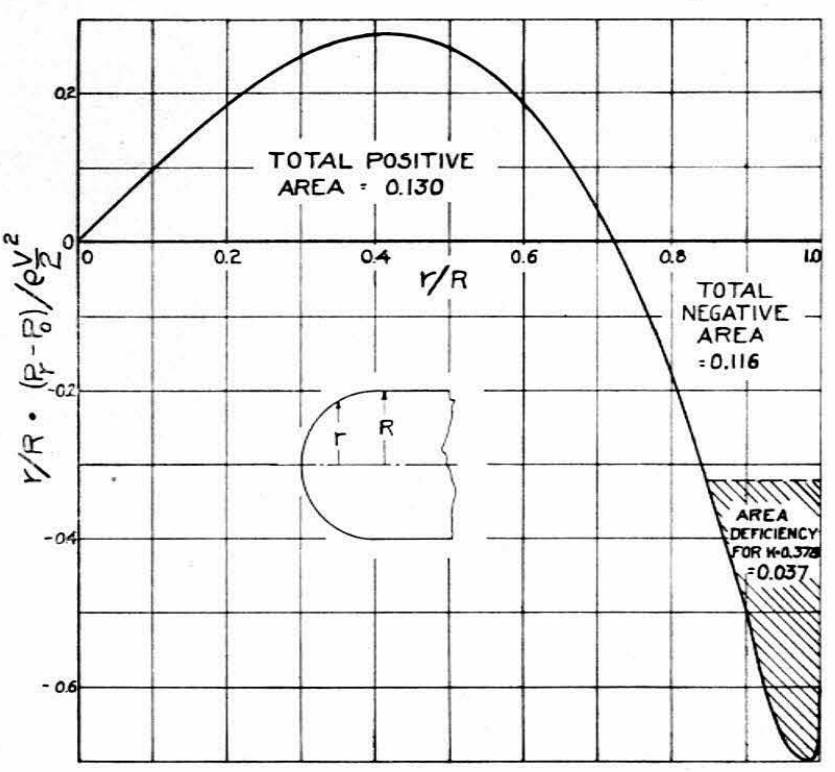

Fig. 3 Axial Force Distribution on Hemispherical Nose

flow thickness from two independent points of view: (a) The cavity cross section and the observed filling time, and $(b)$ the change in the momentum of the re-entrant flow which is equivalent to the increase in drag due to the presence of the cavity.

The momentum calculation is made as follows

$$
\Delta C_{d} A \rho \frac{U^{2}}{2}=M(U+V)
$$

in which

$$
\begin{aligned}
\Delta C_{d} & =\text { change in drag coefficient } \\
A & =\text { cross section of body }=\frac{\pi d^{2}}{4} \\
\rho & =\text { mass density of liquid } \\
U & =\text { velocity of undisturbed flow } \\
M & =\text { mass rate of re-entrant flow }=(\pi d t)(\rho V) . \\
V & =\text { velocity of re-entrant flow }=U \sqrt{1+K} \\
D & =\text { diameter of body } \\
t & =\text { thickness of re-entrant flow }
\end{aligned}
$$

This reduces to

$$
t=\frac{\Delta C_{d} D}{8(1+K+\sqrt{1+K})} \cong \frac{\Delta C_{d} D}{8(2+1.5 K)}
$$

Table 1 gives a comparison of the re-entrant-flow thickness as calculated by the two methods for several different conditions. 
In this table the agreement is surprisingly good, especially considering the difficulties involved in determining experimentally the cavity dimensions. It will be noted that with the exception of the first two examples which are for small-diameter bodies and short cavities, the thickness of the re-entrant flow, as calculated from the experiments, is somewhat greater than the flow predicted from the momentum theory. This is quite possibly due to the ejection of some of the liquid from within the cavity by chance contact of a portion of the re-entrant flow with the highvelocity interface. It also can be interpreted to mean that the
Graphical integration of the axial force on the upstream half of this body gives a drag coefficient of zero since the positive and negative zones agree in area within $1 / 2$ per cent. It is well known that the potential flow for a half-body has zero drag. In this connection Hoerner (16) states, "Half bodies represent the front end of sufficiently elongated bodies with parallel flanks. Positive as well as negative pressures are acting upon the frontal area and the resulting drag is zero, neglecting the small skin-friction forces. This phenomenon of zero drag holds theoretically true for all nose shapes." For this class of nose, the drag due to the presence TABLE 1 COMPARISON OF RE-ENTRANT-FLOW THICKNESS CALCULATIONS.
EXPERIMENTAL OBSERVATIONS VERSUS MOMENTUM THEORY PREDICTIONS

\begin{tabular}{|c|c|c|c|c|c|c|c|c|}
\hline \multirow[b]{2}{*}{$\begin{array}{c}\text { Body } \\
\text { diam, in. }\end{array}$} & \multirow[b]{2}{*}{ Velocity, fps } & \multirow[b]{2}{*}{$K$} & & \multirow[b]{2}{*}{$\begin{array}{c}\text { Cycle time, } \\
\text { sec }\end{array}$} & \multicolumn{2}{|c|}{ Re-entrant-flow } & \multirow{2}{*}{$\begin{array}{l}\text { Thickness } \\
\text { ratio, } \\
(1) /(2)\end{array}$} \\
\hline & & & $\begin{array}{l}\text { Length, } \\
\text { in. }\end{array}$ & $\begin{array}{l}\text { Section, } \\
\text { sq in. }\end{array}$ & & $\begin{array}{l}\text { Experi- } \\
\text { mental (1) }\end{array}$ & $\begin{array}{l}\text { Momentum } \\
\text { theory (2) }\end{array}$ & \\
\hline $1 / 2$ & 90 & 0.241 & 1.0 & 0.046 & 0.0058 & 0.0066 & 0.0099 & 0.67 \\
\hline $1^{2}$ & 90 & 0.348 & 1.0 & 0.0693 & 0.0050 & 0.011 & 0.0131 & 0.84 \\
\hline 1 & 90 & 0.263 & 2.0 & 0.201 & 0.0088 & 0.0188 & 0.0182 & 1.03 \\
\hline 2 & 90 & 0.409 & 1.9 & 0.197 & 0.0075 & 0.0205 & 0.0174 & 1.18 \\
\hline 2 & 90 & 0.378 & & 0.315 & 0.009 & 0.0276 & 0.0231 & 1.19 \\
\hline 2 & 100 & 0.326 & 3.4 & 0.592 & 0.0122 & 0.0352 & 0.0297 & 1.18 \\
\hline 2 & & 0.322 & 3.6 & 0.532 & 0.0136 & 0.0315 & 0.0292 & 1.08 \\
\hline 2 & $77^{1 / 2}$ & 0.334 & 3.2 & 0.444 & 0.0146 & 0.0284 & 0.028 & 1.02 \\
\hline$\overline{2}$ & 59 & 0.326 & 3.0 & 0.523 & 0.0198 & 0.0342 & 0.0297 & 1.15 \\
\hline
\end{tabular}

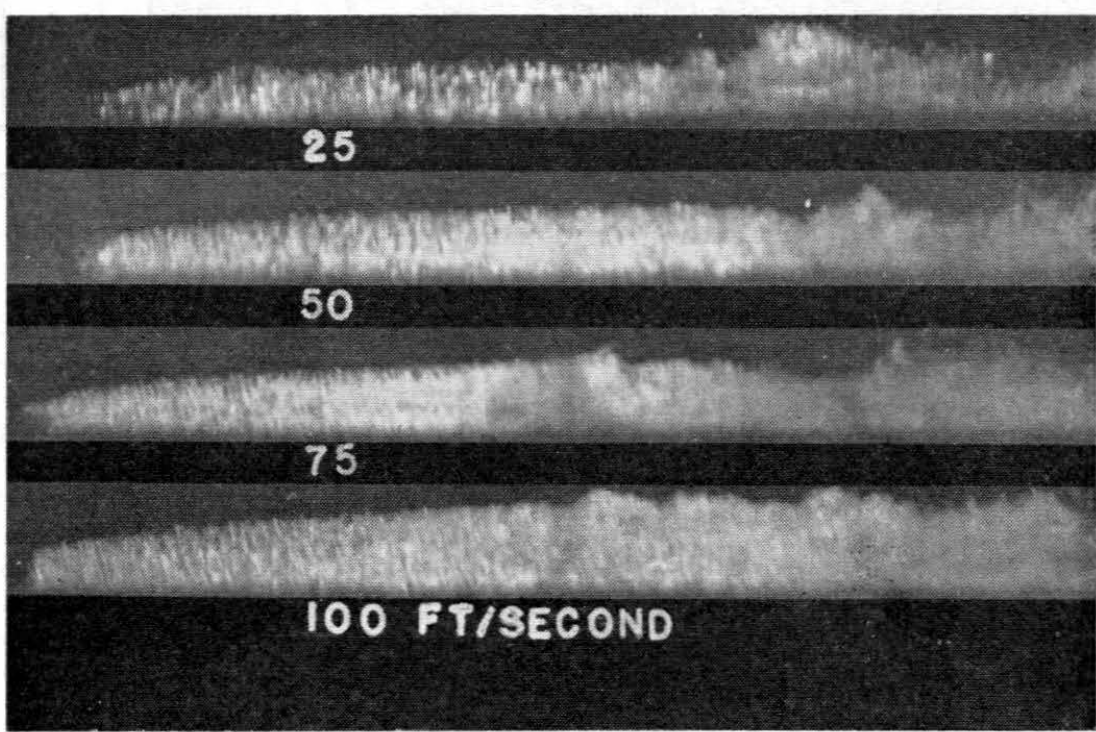

Fig. 4 Effect of Velocity on Traveling-Cavity Size

cavity is not filled completely at breakoff so that the ratio of the two thicknesses indicates the degree of fill, the remainder being uncollapsed or rebound cavities, etc. Six of the nine runs used in the table were made with a tunnel velocity of $90 \mathrm{fps}$. It will be noted that the cycle time varies over a range of nearly $3: 1$. With the same diameter body it increases nearly linearly with the length of the cavity, as would be expected.

The last four runs are on the same body with approximately the same cavity length but with velocities varying from 100 down to $59 \mathrm{fps}$. Here, again, the variation in cycle time is rational as it varies approximately inversely with changes in speed.

It is interesting to note in Fig. 3 that the positive and negative pressure zones on the hemisphere differ in force by only 10 per cent. These are experimental measurements. Landweber (15) used as an example of his method of computing the potential flow about elongated bodies of revolution the rather blunt body whose equation is

$$
y^{2}=0.04\left(1-X^{4}\right)
$$

of the cavity is the total form drag, just as for Gilbarg's completely enveloped body.

If high-speed photographs of fixed cavitation on the same body and same cavitation parameter are compared for a wide range of velocities, it is observed that the average size of the small traveling cavities decreases as the velocity increases. This can be seen in Fig. 4. A possible explanation is that the higher pressure gradients accompanying the higher velocities may produce additional cavities from weaker nuclei than were required for the lower velocities.

Mechanics of Damage. In this phase of the program an attempt has been made to correlate the development of cavitation damage in a flowing liquid with the mechanics of fixed-type cavitation. The damage pattern which developed on the annealed pure aluminum test sections furnished much information about the cavitation-force system and the reaction of the material to the attack. The hardness of the test specimens was very low, averaging 41 Vickers. The yield point is approximately 5000 psi. These low 


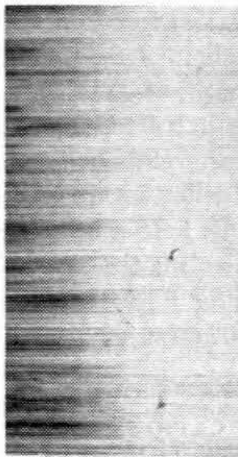

0

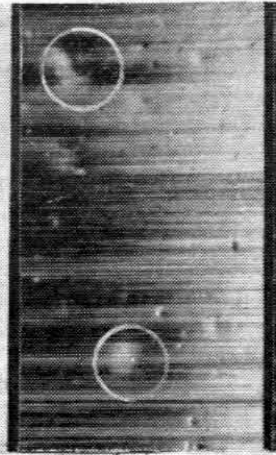

20 MIN.

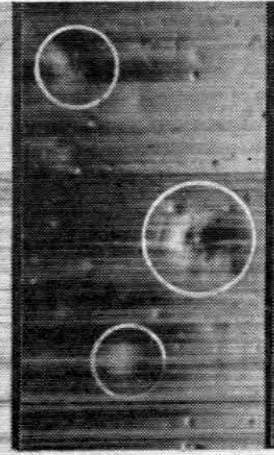

40 MIN.

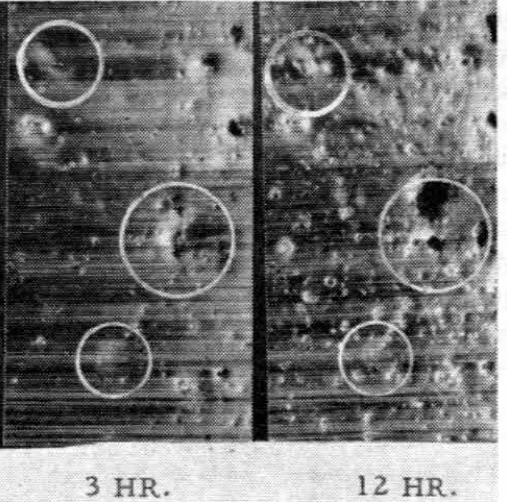

$12 \mathrm{HR}$.

Fig. 5 Damage Development

values are desirable because all blows of sufficient intensity to produce physical damage of any type on the harder materials (bronze, stainless steel, etc.), used in hydraulic equipment where resistance to cavitation is required, will cause permanent deformation in the aluminum. Another useful property of pure aluminum is that the yield point does not change with rate of load application. Thus the same amount of energy will produce the same-size pit independent of the rate at which work is done. Fig. 5 shows damage development on a test section. The five $30-$ diam photomicrographs show the same area for increasing lengths of exposure. Several characteristic features may be observed:

1 The larger pits, such as those in the upper and lower white circles, show elearly that they have been formed by a single blow. Obviously, no metal has been removed, since the toolmarks can be seen, undiminished in sharpness, over the total area of the pit. Smaller pits appear to be formed in the same manner.

2 After $20 \mathrm{~min}$, the pits are widely spaced with no other visible surface damage. This means that the cavitation developed only a few blows of damaging intensity during this long period.

3 If the micrographs for the longer periods are compared to the 20-min one, many of the original pits are found unchanged; the main difference is that new pits appear on the intervening surfaces. In some cases, e.g., in the large center circles, the pits have changed, apparently due to overlapping hits or "near misses."

Note that this damage was produced by cavitation in cold water at the relatively high velocity of $90 \mathrm{fps}$. This is well above the minimum velocity for which cavitation damage has been observed in many different metals in hydraulic equipment. Pure aluminum in the annealed condition has a very low hardness and yield point.

Frequency of Damaging Blows. The frequency of damaging blows is obtained by counting the number of pits, using a modified "blood-count" technique. The pits were classified roughly according to diameter: i.e., small, below 0.0025 in.; medium, 0.0025 to 0.005 in.; large, 0.005 to 0.01 in.; extra large, above 0.01 in.

Fig. 6 shows pitting rate versus exposure time for eight 1-in. cavity test sections and one 2 -in. cavity. The numbers on the points are the test-specimen identification numbers. The velocity was constant. Although the range of exposure time is large, the number of pits appearing per unit time is about constant. It tends to decrease with-increasing time, largely because duplicate hits escape detection. Motion pictures taken of the cavity surface during these runs were measured to determine the average size and "packing" of the traveling cavities. The cavities

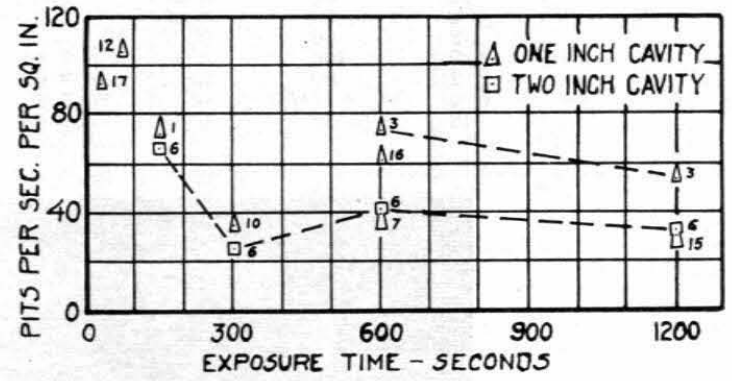

Fig. 6 Effect of Exposure Time on Pitting Rate

averaged 0.025 in. diam. Approximately $1.8 \times 10^{6}$ cavities per sec per in. of circumference were swept into the stagnation zone. Fig. 6 is for the maximum damage zone.

Figs. 9 and 10 show that the rate of pitting drops rapidly on both sides of the maximum. The summation over the 3-in. section is 60 pits per sec per in. of circumference. Hence only one in 30,000 of the traveling cavities causes a damaging blow. The question is - what happens to the other 29,999 ? The present guess is that the high-pressure zone above the surface acts as a sorting screen. The smaller cavities collapse so far away that the resulting shock wave is attenuated to below the damaging intensity before it reaches the surface. Everything favors the large cavities-higher collapse pressures with longer collapse times which earry them closer to the surface. This concept implies that liquid properties such as the size and number of the nuclei $(17,18)$ are of great importance, and may control the degree of damage for an otherwise constant cavitation condition.

In the zone of maximum damage an average of 56 pits, each with a mean area of $0.000003 \mathrm{sq}$ in., are formed per sec per sq in. Thus each surface element is struck only once every $100 \mathrm{~min}$ or, assuming 24 -hr operation, 5000 times per year. This low rate does not appear consistent with the commonly accepted concept that cavitation damage is essentially a fatigue failure caused by a large number of light blows. This force system seems more compatible with the concept that each blow produces permanent deformation and damage.

Intensity of Individual Cavitation Blows. It is possible, by using broad assumptions, to calculate the initial size of the collapsing cavity required to produce a damage pit. The Vickers diamondpyramid hardness is obtained by measuring the dimensions of the indentation produced in the material by a known load. On the aluminum test sections the sizes of the hardness indentations and the damage pits are of about the same order, and the base-todepth ratios are also comparable. The work done per unit 


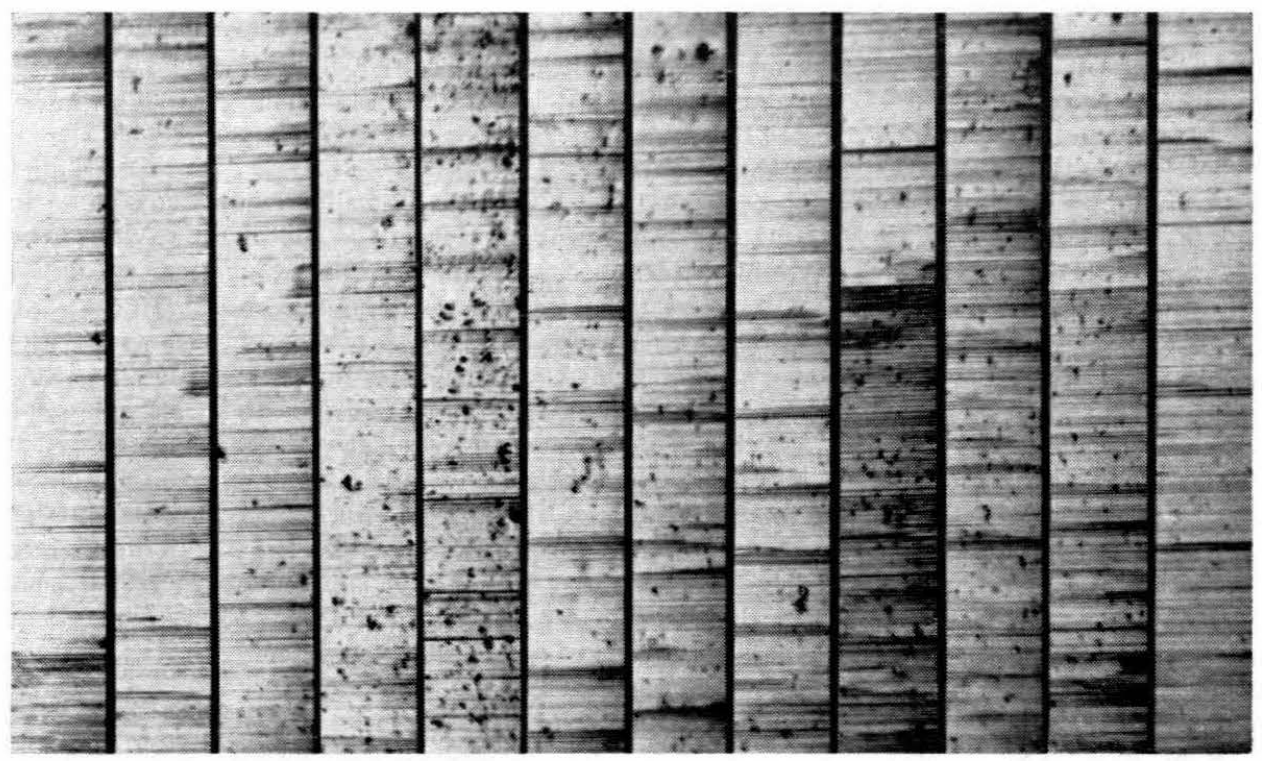

Fig. 7 Photomicrographs of Pitting With 1-In. Cavity
Fig. 8 PhotomicRographs OF Pitting With 2-In. Cavity

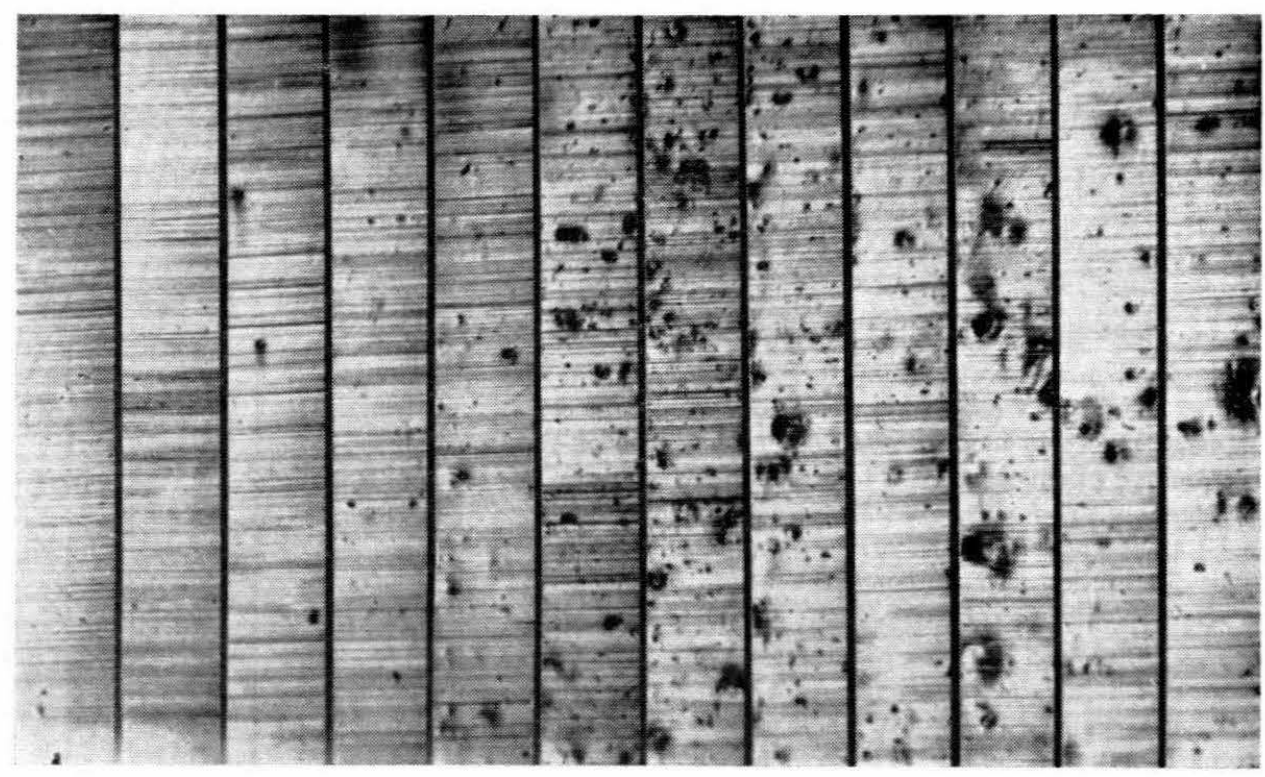

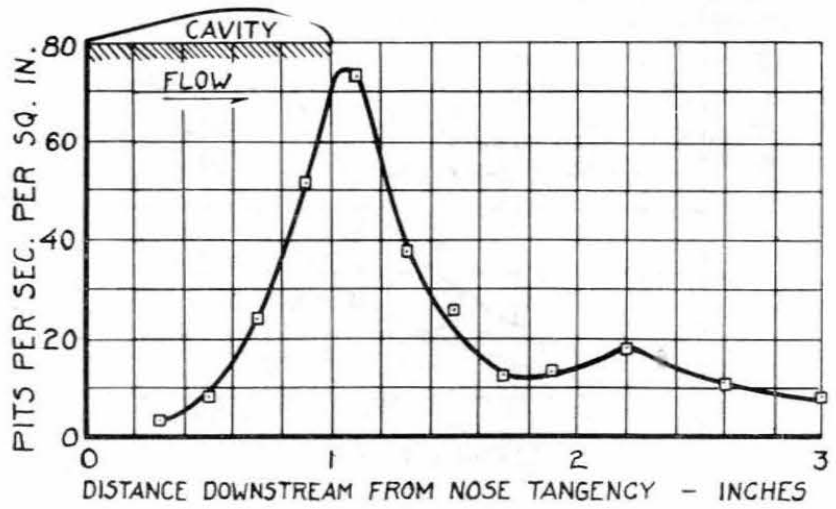

Fig. 9 Distribution of Pitting for 1-In. Cavity Length

volume of indentation is easily calculated from the dimensions of the indentation and the known load applied by the hardness tester. It is about $60,000 \mathrm{in}-\mathrm{lb}$ per $\mathrm{cu}$ in. Assuming the damage

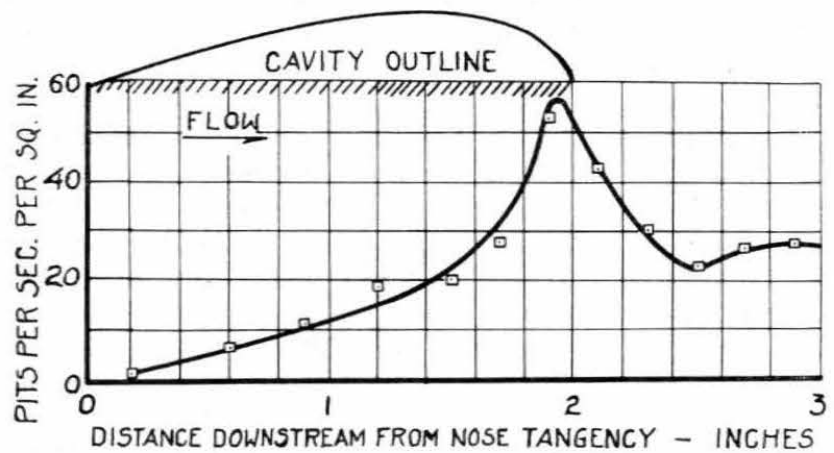

Fig. 10 Distribution of Pitting for 2-In. Cavity Length

pits are spherical segments, their volumes may be calculated from their diameters and depths. The work done in forming the pit is the product of its volume by 60,000 , assuming that the work 
of formation per unit volume is the same for the damage pit and for the hardness indentation.

The fraction of the total energy radiated from a collapsed cavity used in producing a pit may be assumed to be the ratio of the volume of the spherical pyramid whose base is the pit surface, to the volume of this sphere. Thus the required energy of the pit-forming eavity is obtained by dividing the energy used to produce the pit by this ratio. The diameter of this cavity is now calculable, as the work done $(P V)$ by the fluid during collapse must equal the collapse energy. Since collapse must start as soon as the local pressure rises above vapor pressure, a reasonable estimate of the average pressure causing collapse is half the stagnation pressure. The results of such calculations for three typical damage pits are given in Table 2. These calculated cavities are considerably larger than the average cavities seen on the interface. This is consistent with the conclusions of the previous section.

Distribution of Damage. Fig. 7 shows strips from the 12 photomicrographs of a test section after a 10-min exposure to a 1 -in. cavitation zone. If these strips were each rotated counterclockwise $90 \mathrm{deg}$ and placed end to end, they would be in their true relative position, with flow from left to right. Note the toolmarks. zone is approached. Further investigation is needed to evaluate the relative importance to the production of damage of these opposing variables with cavity length, i.e., the lower pitting rate in the maximum damage zone versus the larger average size of pits.

Figs. 9 and 10 both indicate a small secondary maximum in pitting rate just downstream from the end of the cavity. The cause of this is not clear. One possible explanation, which has some confirmation from the photographic record, is that this increase may be due to the recollapse of some of the larger cavities which may rebound after first collapse in the stagnation zone.

Effect of Velocity on Cavitation Intensity. In the consideration of the results of this series of tests it must be remembered that it is a preliminary investigation consisting only of one small series of runs. Furthermore, it, like the rest of the experiments reported herein, applies only to fixed-type cavities. Finally, in the analysis of these tests, intensity of cavitation is assumed to be proportional to rate of pitting, modified by qualitative observations as to changes in the proportion of large pits produced.

Fig. 11 shows the observed pitting rate as a function of velocity. The circled points give the average rate of pitting over the entire length of the specimen. The crosses show the pitting rate in the

TABLE 2 INITIAL CAVITY DIAMETER AND COLLAPSE CENTER LOCATION PREDICTED FROM DAMAGE-PIT DIMENSIONS

\begin{tabular}{cccc}
\hline \multicolumn{4}{c}{ Damage pit- } \\
Work of & $\begin{array}{c}\text { Volume } \\
\text { ratio }\end{array}$ \\
0.005 & 0.0007 & 0.00042 & 0.077 \\
0.007 & 0.0006 & 0.00052 & 0.029 \\
0.0024 & 0.0004 & 0.000055 & 0.10
\end{tabular}

\begin{tabular}{|c|c|c|}
\hline $\begin{array}{c}\text { Work of } \\
\text { collapse, in-lb }\end{array}$ & $\begin{array}{l}\text { Iñitial } \\
\text { diam, in. }\end{array}$ & $\begin{array}{l}\text { Collapse } \\
\text { center to } \\
\text { surface, in }\end{array}$ \\
\hline 0.006 & 0.073 & 0.005 \\
\hline & 0.106 & \\
\hline 0.00054 & 0.033 & 0.002 \\
\hline
\end{tabular}

In Fig. 9 the pitting rate is plotted against position along the test section. The relative size and location of the fully developed cavity is outlined at the top of the diagram. The maximumdamage zone coincides with the stagnation zone at the downstream end of this cavity. The pits above and below this zone presumably are formed during the sweeping of the stagnation zone along the full length of the test section during breakoff. This distribution seems in good accord with the concept of fixedcavity mechanics presented herein.

Figs. 8 and 10 are from a similar cavitation run for a 2 -in. cavity. One striking feature is the lower pitting rate in the maximum-damage zone. However, the average pitting rate over the entire length of the test section is nearly identical for both cavity lengths, being 59 pits per sec for the 1-in. cavity and $60^{1 / 2}$ pits per sec for the 2 -in. cavity. This agreement is also consistent with the fixed-cavity mechanics since the rate of traveling-cavity formation on the first cavity interface is primarily determined by the velocity and liquid properties and not the cavity length.

These findings imply that the total amount of damage, as measured by the material removed, may be relatively independent of the cavity length, whereas the maximum-damage intensity may be produced by very short cavities. On the other hand, a comparison of Fig. 8 with Fig. 7 points out a difference with length; i.e., although the number of pits on the two specimens was nearly identical, the 2-in. cavity produced a higher proportion of large pits. This implies that a higher percentage of large traveling cavities forms on the interface of the longer fixed cavity. Two factors seem to favor this, i.e., a slightly lower absolute pressure and a much longer time between inception and collapse. Probably the latter is the more important, for although, as previously stated, most of the growth in diameter of the traveling cavities takes place at the upstream end of the fixed cavity, there is some indication that slow growth continues until the high-pressure

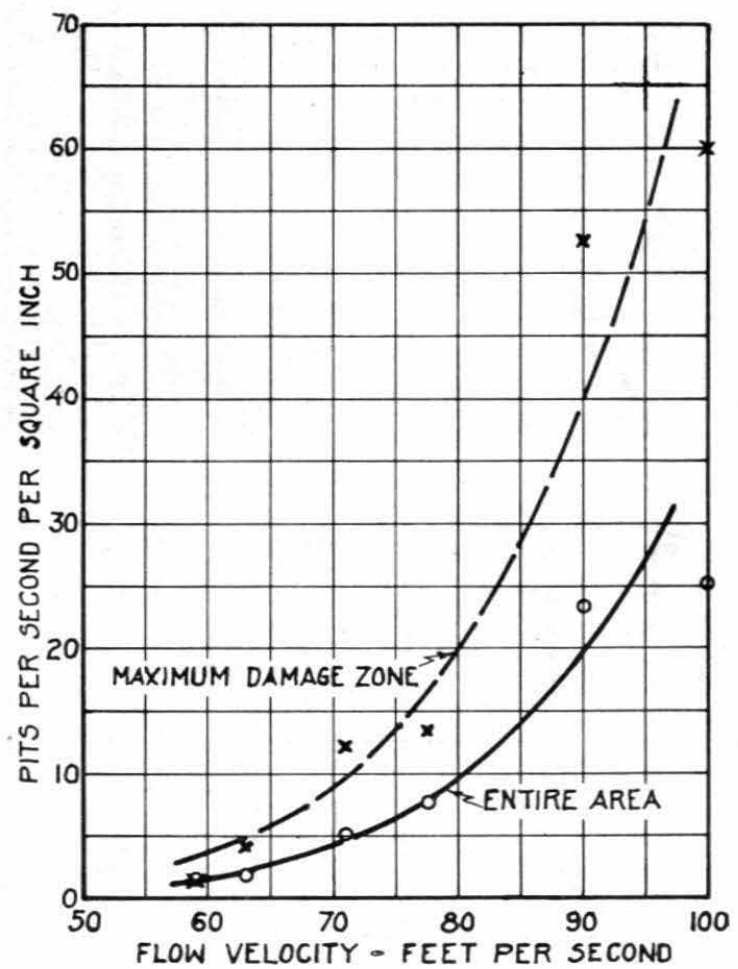

Fig. 11 Variation of Pitting Rate With Flow Velocity

maximum-damage area at the nominal end of the cavity. There is considerable scatter in the results. It is believed that this is due chiefly to variation in hardness between the test sections. A subsequent investigation showed that the time of anneal had not 
been adequate to insure uniform properties of the different specimens. In spite of this scatter it is very clear that the rate of pitting falls very rapidly with decrease in speed.

Fig. 12 shows the same results plotted on logarithmic coordinates. For the 90 and $100-\mathrm{ft}$ velocity rate the points plotted are the average of the different specimens tested. It will be seen that the points fall on the straight lines which best fit the two sets of points. Both have steep slopes, approximately six. This indicates that the pitting rate varies roughly with the 6 th power

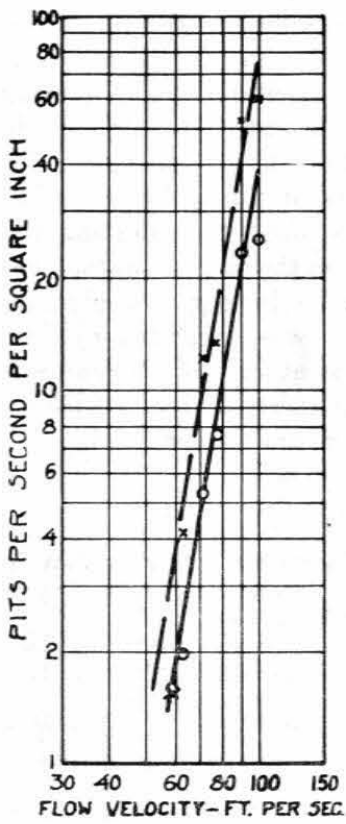
gram of Pitting Rate Versus Flow Velocity
Fig. 12 Logarithmic Diaof the velocity. This is, of course, a tremendous rate of change. The evidence, however, is too meager to justify more than speculations concerning the implications.

Sixth-Power Variation of Pitting Rate With Velocity. The 6th-power variation implies that the pitting rate would about double for each $12^{1 / 2}$ per cent increase in local velocity, assuming all other conditions, including size and location of the cavity, remain the same. Such a high sensitivity to the local velocity would help to explain some of the apparent discrepancies in the amount of cavitation damage observed on similar machines operating under nearly the same field conditions. Another type of implication carried by these results is the great importance of using a carefully standardized and controlled cavitation intensity in the testing of the relative resistance of materials.

The reason for this high sensitivity of the cavitation intensity to change in velocity is not obvious. Since the cavity size remained constant throughout these experiments, the geometry of the flow was unchanged. Hence the velocity distributions at any cross section were similar for all speeds. However, the base pressure, i.e., the cavity pressure, has the same absolute value for all the runs, i.e., the vapor pressure of the liquid. The maximum pressure rise above this base was equal to the velocity head of the flow along the interface, which is proportional to the square of the average velocity. In fact, the entire pressure field will vary with the square of the average velocity. Now, Rayleigh's derivation of the time of collapse of a spherical cavity shows that this time is inversely proportional to the square root of the pressure difference. Since this pressure difference is proportional to the square of the flow velocity, the time of collapse will vary inversely with the velocity, or since the distance is proportional to the product of the velocity and the time, the length of path required to collapse the cavity will not vary with the velocity. This means that for all velocities the cavities will collapse in the same position relative to the surface. Therefore the great increase in pitting rate cannot be explained on the basis that the average distance from the surface to the center of collapse would be less with the higher velocity. An increase in pitting rate will be anticipated if the average cavity size increased with velocity. Fig. 4, however, shows that, in general, the average size of the cavity appears to decrease slightly with increase in velocity. This should tend to decrease rather than increase the pitting rate.

Attempts to measure the average size of cavity from these pictures show that the variations with velocity are too small to be distinguishable within the available definition. Thus it can be said that, within the accuracies of these experiments, the cavity size remains sensibly unchanged with changes in velocity. Since the energy of collapse is the product of the initial volume of the cavity and the pressure causing collapse, this energy will increase with the square of the velocity. Therefore, as the velocity increases, a given-size cavity collapsing at a given point will produce a larger pit and also cavities collapsing at a greater distance from the surface will be able to produce pits.

Another factor which should increase the rate of pitting is that the number of cavities swept into the collapse zone increases linearly with the velocity. At first sight it would appear that the combination of these two factors should increase in pitting rate with the cube rather than the 6 th power of the velocity. However, since the number of cavities swept into the collapse zone for each pit is so great, it appears certain that a very small increase in the radius of effective action of the collapsing cavities would greatly increase the number of cavities able to produce pits. At the present time these three factors seem to offer the most acceptable explanation of the high sensitivity of the rate of pitting to change in velocity.

Pit Size-Distribution. In the discussion of the method of counting the pits, it was explained that, during the counting, the pits were classified according to diameter into four groups, with the three larger groups each covering a range of $2: 1$ in diameter and with the smallest group consisting of all those pits below 0.0025 in. diam. On the previous runs, which were all at $90 \mathrm{fps}$, it was observed that over 95 per cent of all pits were in the smallest group. An examination of the counts for the speed runs shows that the percentage of pits in the three larger classes varies with velocity as follows:

$\begin{array}{cc}\text { Ft per sec } & \text { Per cent } \\ 100 & 5.1 \\ 90 & 4.1 \\ 77^{1} / 2 & 2.6 \\ 71 & 2.65 \\ 63 & 0 \\ 59 & 0\end{array}$

These results do not justify plotting, but it appears probable that the percentage of large pits also increases with some large power of the velocity; hence it may well be that the damage rate, as measured by rate of removal of material, will be found to vary even more rapidly than the 6 th power of the velocity.

\section{Summary and Conclusions}

Fixed-type cavitation appears to be one of the most prevalent forms of cavitation occurring in flowing liquids. The idealized concept of fixed cavitation includes no mechanism for producing damage. A study of the real phenomenon shows that a multitude of small cavities sweep along the interface of the fixed cavity and collapse in the downstream stagnation zone. Thus the mechanism of damage is similar to that for the simpler traveling type of cavitation. The small size of the traveling cavities in the fixedtype cavitation is apparently counterbalanced by the high pressure available to produce collapse in the stagnation zone. One important feature of the fixed cavity is re-entrant flow at the downstream end. This upstream flow rapidly fills the cavity and is the primary cause of the characteristic growth-filling-breakoff cycle. Because of this cyclic process the stagnation zone sweeps the entire cavity length and the downstream region as well, thus creating relatively large areas of moderate cavitation damage on both sides of the maximum-damage zone.

Much significant information about the type and intensity of the cavitation attack can be derived from the pitting pattern on soft metallic specimens. One surprising fact is the relatively low 


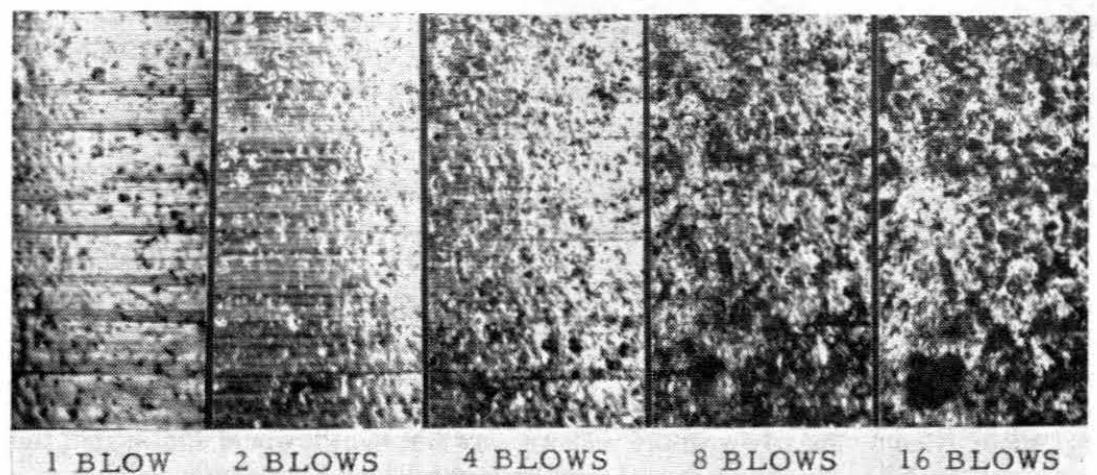

Fig. 13 Damage Development in Maximum Damage Zone

vestigate the pitting rate on bodies of the same size but with different nose shapes to see the effect of this variable and whether it can be isolated from that of velocity of flow and cavity length.

\section{Acknowledgments}

The primary support for the work described herein was received from Contract Nonr$220(08)$, which contains funds from the Office of Naval Research and the Bureau of Ships of the U. S. Navy. Selection of aluminum as the material for the damage test sections was suggested by $\mathrm{Mr}$. W. S. Rheingans, who has shown continuing interest in all aspects of the author's cavita-

pitting rate. Thus the annealed aluminum used in this study is soft enough to pit under an applied force of much lower intensity than the fatigue limits of the materials used in the construction of hydraulic equipment. Nevertheless, at $90 \mathrm{fps}$, which is well above the lower limit for cavitation damage on these materials, a given point on the surface is hit only once every $100 \mathrm{~min}$. This leads to the tentative conclusion that damage produced by fixedtype cavitation is caused by relatively infrequent high-intensity blows which either remove particles of material by each blow or by early fatigue. It will be recalled that, on the basis of the pitting rate and the average diameter of the pits, it was calculated that each point on the surface would, on the average, be struck once every $100 \mathrm{~min}$. By chance, the time intervals used in one damage run have a 90 -min base, corresponding roughly to the average time between blows. Thus the five photomicrographs in Fig. 13 show the "blow-by-blow" damage to a typical area in the maximum-damage zone.

In the past a basic obstacle to the study of the mechanics of cavitation damage has been the lack of any measure of intensity of cavitation. The discovery that fixed cavitation produces damage blow by blow, and that the repetitive rate is relatively low, suggests the use of a soft metal brought to a standardized physical condition for a working measure of eavitation intensity. For this use the specimen should be exposed to the cavitation for only a few minutes to avoid superposition of pits. The determination would be made by measuring the size range and rate of formation of the pits in place of the usual loss-of-weight measurement. The damage-resistant properties of the soft test specimen are not relevant. This material would only be used to obtain a record of the intensity and frequency of all of the blows capable of damaging, in any manner, the metal structure of the equipment under consideration.

One significant example of information obtainable by this approach is the fact that the total number of damaging blows struck in a cavitating region in a given time seems primarily a function of the physical properties of the liquid and the relative velocity of flow. Reasonable changes in the cavity length, with corresponding changes in the cavitation parameter, do not alter the total number of blows struck. Thus further study may show that total damage is independent of the length of the cavitation zone and intensity of damage may be greatest for short cavities.

Additional work needs to be done to clarify the effect of velocity variation on pitting rate and also to correlate pitting rate and pit size on standardized test specimens with damage as measured by rate of removal of material. Also, it begins to appear important to investigate the variation in intensity of cavitation with the size and shape of the cavity. Preparations are now under way to study pitting rate on a series of geometrically similar bodies of the same general shape as those reported herein, but varying in size over a relatively wide range, and also to in- tion research program. The principle used in estimating the stream thickness of the re-entrant flow on the basis of the momentum required to balance the deficiency in negative pressure caused by the presence of the eavity was suggested by $\mathrm{Mr}$. Joseph Levy of the Hydrodynamics Laboratory staff. The author also gratefully acknowledges the help received from his assistants, Frank Bonamassa, James Isaacson, and Salyer Reed.

\section{BIBLIOGRAPHY}

1 "An Annotated Bibliography of Cavitation," by F. A. Raven, A. M. Feiler, and Anna Jespersen, Navy Department, David Taylor Model Basin, Washington, D. C., Report R-81, 1947.

2 "Untersuchungen uber Kavitation und Korrosion bei Turbinen, Turbopumpen und Propellern," by Dr.-Ing. Hermann Föttinger, "Hydraulische Probleme," 1926, pp. 14-64, 107-110.

3 "On the Mechanism and Prevention of Cavitation," by Phillip Eisenberg, Navy Department, David Taylor Model Basin, Washington, D. C., Report 712, 1950.

4 "A Brief Survey on the Progress of the Mechanics of Cavitation," by Phillip Eisenberg, Navy Department, David Taylor Model Basin, Washington, D. C., Report 842, 1953.

5 "Water Tunnel Investigations of Steady State Cavities," by H. L. Pond and Phillip Eisenberg, Navy Department, David Taylor Model Basin, Washington, D. C., Report 668, 1948.

6 "Cavitation Characteristics and Infinite-Aspect-Ratio Characteristics for a Hydrofoil Section," by J. W. Daily, Trans. ASME, vol. 71,1949 , p. 269.

7 "Kavitation und Kavitationskorrosion," by J. Ackeret, "Hydromechanische Probleme des Schiffsantriebs," 1932, p. 227.

8 "Werkstoffzerstörung durch Kavitation," by Hans Nowotny, VDI-Verlag, Berlin, Germany, 1942, 84 pp.

9 "Accelerated Cavitation Erosion," by W. S. Rheingans, Trans. ASME, vol. 73, 1950, p. 705.

10 "On the Pressure Developed in a Liquid During the Collapse of a Spherical Cavity," by Lord Rayleigh, Philosphical Magazine, series 4 , vol. 34,1917 , pp. 94-98.

11 "Laboratory Investigations of the Mechanism of Cavitation," by R. T. Knapp and A. Hollander, Trans. ASME, vol. 70, 1948, p. 419. 12 "Cavitation Mechanics and Its Relation to the Design of Hydraulie Equipment," by R. T. Knapp, James Clayton Lecture, Proceedings of The Institution of Mechanical Engineers, series A, vol. 166,1952 , pp. $150-163$.

13 "Free Boundaries and Jets in the Theory of Cavitation," by David Gilbarg and James Serrin, Journal of Mathematics and Physics, vol. 29, April, 1950, pp. 1-12.

14 "Cavitation and Pressure Distribution," by Hunter Rouse and J. S. MeNown, Bull. 32, No. 42, State University of Iowa, 1948. 15 "The Axially Symmetric Potential Flow About Elongated Bodies of Revolution," by L. Landweber, Report 761, David Taylor Model Basin, Navy Department, Washington, D. C., August, 1951.

16 “Aerodynamic Drag," by S. F. Hoerner, 1951, published by the author, 148 Busteed, Midland Park, N. J.

17 "Cavity Formation in Water," by E. N. Harvey, W. D. McElroy, and A. H. Whiteley, Journal of A pplied Physics, vol. 18, February, 1947, p. 162.

18 "The Effect of Air Content on the Appearance of Cavitation in Distilled, Salt, and Sea Water," by F. Numachi and T. Kurokawa, Translation and Commentary, Ordnance Research Laboratory, Pennsylvania State College State College, Pa., 1946. 